\title{
CAMBIOS EN EL CONSUMO DE ALCOHOL EN ADOLESCENTES ESCOLARIZADOS DE LA LOCALIDAD DE SUBA ENTRE LOS AÑOS 2010 Y 2015
}

\author{
Tutor de la investigación: Juan Carlos González Q.** \\ Blanco Hernández Andrés Felipe, Borras Astro Juan Camilo, Camacho Lozano Johandra Viviana, \\ Camacho Solano Karen Andrea, Camargo Vargas Diego Alexander ${ }^{\dagger+}$.
}

\begin{abstract}
RESUMEN
Objetivo: Comparar los resultados obtenidos con un estudio sobre el consumo de alcohol en adolescentes realizado en 2010, para determinar si ha aumentado, disminuido o continuado igual dicho consumo e identificar, por medio de las variables indagadas, factores de riesgo y protectores.

Metodología: Se aplicó una encuesta personalizada a adolescentes de ambos sexos, entre las edades de 10 y 20 años pertenecientes a una Institución Educativa Distrital (IED) de la localidad de Suba (Bogotá) en el año 2015, indagando con variables que pudiesen modificar el consumo de alcohol en adolescentes. Resultados: Según los resultados encontrados se puede afirmar que en el transcurso de cinco años, del 2010 al 2015, el consumo de alcohol en la población estudiada ha disminuido en un tercio aproximadamente, con una significancia estadística importante y, que el consumo de alcohol de manera frecuente ha descendido también. Por otra parte, no se encontró diferencia en algunos factores de riesgo tales como el que los padres o los amigos y compañeros consuman alcohol, por lo que se podría afirmar que, en este estudio, dichos factores no están afectando de la misma manera las decisiones respecto al inicio de consumo y mantenimiento de esta práctica.
\end{abstract}

Palabras clave: Adolescente, alcohol, consumo de bebidas alcohólicas, factores de riesgo, prevención.

\begin{abstract}
Objective: To compare the results of this study with a previous one about alcohol consumption in adolescents carried out in 2010. The objective is to determine if the consumption has been increased, reduced or remained as in 2010. Also to identify factors of both risk and protection for consumption through the studied variables.

Methodology: A personalized survey was carried out on adolescents ranging in ages from 10 to 20 years, who attending a public school from a locality in Bogotá in 2015. The survey used variables that might modify the alcohol consumption in adolescents.

Results: According to the obtained results, it could confirm that the alcohol consumption decreased in about one third of the studied population, showing an important statistical difference. Also the drinking frequency of alcoholic beverages has been reduced. Risk factors such as the consumption by parents, friends, and peers showed no difference. This last fact allows to affirm that these factors are not affecting the decisions about the consumption start and continuity of this practice.
\end{abstract}

Keywords: Adolescent, alcohol, alcohol drinking, risk factors, prevention.

\section{INTRODUCCIÓN}

\section{Contexto}

El consumo abusivo de alcohol es un problema de salud pública que se ha relacionado con diferentes causas. Estudios recientes informan el papel que juegan los factores hereditarios en interacción

** MD. Especialista Medicina Familiar Integral, MSP. Director Departamento de Medicina Comunitaria juan.gonzalez@juanncorpas.edu.co

${ }^{++}$Estudiantes VII semestre de Medicina. Fundación Universitaria Juan N. Corpas.

Carta Comunitaria. Vol. 23. Número 135. Nov. - Dic. 2015 
medioambiental (1). El alcohol es la sustancia psicoactiva más usada a nivel mundial (2). El consumo de sustancias se inicia en la adolescencia, en muchos casos con aquellas de fácil acceso como el alcohol y la nicotina (3), debido a que esta es una etapa que tiene mayores niveles de fragilidad emocional, vulnerabilidad y disposición a dejarse influenciar por un grupo social (4). En adolescentes escolarizados se han estudiado diferentes variables que influyen en el consumo temprano de alcohol, relacionadas con factores individuales, escolares, familiares y sociales (5). Dentro de cada una de estas variables se revisan diferentes condiciones que promueven el consumo de alcohol en la adolescencia. Las condiciones individuales son al edad (2), por las características ya descritas en esta etapa; en el componente emocional es posible que el alcohol sea utilizado como una forma de afrontar un estado de ánimo disfórico y está demostrado que se aumenta el consumo cuando coexisten estados depresivos con la búsqueda de nuevas experiencias (1).

\section{Factores de riesgo}

El consumo de alcohol se ve condicionado por la presencia de este hábito en familiares con quienes se convive (2), por la carencia de apoyo familiar que conlleva al consumo de sustancias como una alternativa para enfrentar el estrés generado por el ambiente $(5,6)$ y la desintegración familiar (2). Los problemas familiares son señal de riesgo para el inicio y progresión del consumo (4). En el contexto escolar se pueden relacionar el nivel educativo con el consumo de alcohol y, asimismo, con la disminución en el rendimiento académico $(1,4)$, como lo señala Rodríguez en sus estudios, el consumo reciente está directamente relacionado con la capacidad cognitiva (2).

\section{Inducción hacia el alcohol}

En la actualidad, las bebidas alcohólicas son de fácil acceso y tienen aceptación social. El adolescente está en una etapa de exploración de lo que se encuentra en su entorno. La yuxtaposición de estas circunstancias aumenta la incidencia del consumo (5). Adicional a esto, la publicidad atractiva brindada por los medios de comunicación incrementa el consumo (2). La incidencia del consumo se relaciona con la aceptación social, "según las normas de comportamiento de los pares, se influencian los comportamientos propios por un proceso de comparación social que sirve como estándar para evaluar los comportamientos y ser visto como similar al grupo de referencia" (4). A su vez, la interacción entre pares se asocia con el mantenimiento y progresión del consumo (2).

Teniendo en cuenta las variables mencionadas existen estrategias protectoras que aspiran disminuir la incidencia del consumo. En estudios realizados se han encontrado algunos factores que tienden a proteger al adolescente, tales como la influencia positiva del grupo de amigos, la adecuada comunicación entre padres e hijos, el soporte emocional y la compañía de la familia y, la religión (2). Un estudio descriptivo transversal realizado por Álvarez (6) demostró que una elevada autoestima disminuye el riesgo del consumo (2). El estado colombiano ha expedido leyes como la 124/1995, 232/1995, 1335/2009, el Código Nacional de la Policía y el Decreto 1375/70, en los cuales se prohíbe el consumo de cigarrillo y el alcohol a los menores de edad (4); no obstante, a pesar de los intentos de prevención, la concepción social y mental del alcohol por parte de los adultos disminuye la precepción del riesgo de consumirlo (5).

\section{EI asunto hoy}

Actualmente el consumo de alcohol es una problemática social que se inicia a muy temprana edad (2), siendo el promedio 16 años (7) y cada año va disminuyendo la edad de inicio de consumo (4), con una prevalencia mayor en el sexo masculino, según el segundo Estudio de Salud Mental del Ministerio de Salud, el cual determinó que un 13,2 \% de los hombres abusa del alcohol contra un 1,6\% de las mujeres y la dependencia fue de $4,7 \%$ en los hombres y el 0,3\% en mujeres en Bogotá (8), aunque 
se evidencia en otros estudios que las mujeres están mostrando igualdad de consumo con los hombres (1).

El alcohol es la puerta de entrada para el consumo de otras sustancias psicoactivas e ilegales que generan mayores consecuencias (3), por esto es importante intervenir en los factores de riesgo y promover los factores protectores.

\section{La intención de esta investigación}

Con esta investigación se quiso identificar cada uno los factores familiares, sociales y socioculturales que influyen -ponen en riesgo o protegen a los adolescentes escolarizados- en el inicio y el mantenimiento del consumo de alcohol $y$, al mismo tiempo, compararlos con los resultados encontrados en la investigación realizada en 2010 e identificar cuáles han sido los cambios que se han presentado durante estos cinco años. Es importante realizar esta investigación porque el consumo de alcohol en esta etapa de la vida se asocia a comportamientos antisociales (actos violentos, conductas problemáticas, etc.); además, el consumo de alcohol afecta la parte cognitiva del adolescente, tiene consecuencias en la facultad de la memoria, la capacidad de aprendizaje y otras funciones cognitivas importantes (9). Dado lo anterior, se hace necesario identificar los factores de riesgo para poder realizar alguna intervención en ellos buscando su disminución. De igual manera, determinar los factores protectores para fortalecerlos y ayudar al adolescente a evitar conductas que sean perjudiciales para su salud física o mental, a corto o largo plazo.

\section{METODOLOGÍA}

Tipo de estudio: Cuantitativo, observacional de tipo corte.

Población: Se contó con la participación de adolescentes de ambos sexos entre las edades de 10 y 20 años de la cuidad de Bogotá pertenecientes a una Institución Educativa Distrital de la localidad de Suba.

Criterios de inclusión: Jóvenes de todos los grados académicos de secundaria de una Institución Educativa Distrital de la localidad de Suba. A los adolescentes de menor edad y a los que tenían alguna dificultad de aprendizaje o algún déficit cognitivo se les fue leyendo y explicando cada una de las partes de la encuesta para que la entendieran correctamente.

Variables: Se utilizó una encuesta, previamente empleada por el Programa Integral de Prevención de Salud del Adolescente (PIPSA), que mide las características de frecuencia y cantidad de alcohol que consumen los adolescentes escolarizados y las diversas situaciones en las cuales el consumo involucra una respuesta a un evento de naturaleza psicológica o física, integrando emociones desagradables, malestar físico, emociones agradables, necesidad o urgencia por consumir alcohol.

Las variables usadas en la encuesta fueron: ¿Durante la última semana ha bebido (cerveza u otra bebidas con alcohol)? y ¿cuántas veces? Variables del test de Cage: ¿Ha tenido dificultades porque consume drogas o bebidas alcohólicas en la escuela o en su casa?, ¿se ha hecho daño o le ha hecho daño a otra persona accidentalmente estando bajo el efecto del alcohol o las drogas?, ¿ha comenzado a consumir mayores cantidades de drogas o alcohol para obtener el efecto que desea?, ¿a qué edad probo el alcohol? y variables destinadas a estimar influencias para beber alcohol tales como: ¿Alguno de sus padres "bebe" o consume drogas?, ¿alguno de sus amigos (con los que usted pasa mucho tiempo) "bebe" o consume drogas?, ¿cuánto bebe en la actualidad? y si le gusta. Se hicieron las mismas preguntas del estudio de 2010 a una población en similares circunstancias. 
Recolección de información: Se presentó ante las directivas del centro educativo la investigación, logrando su aprobación y ejecución durante los meses de febrero y marzo de 2015 . Una vez en los salones, se explicó la encuesta y la voluntariedad para participar. Se insistió en que si no querían responder alguna pregunta, podían dejar de hacerlo, solicitando que aquellas preguntas que respondieran se hicieran con la verdad. Se pidió a los profesores que salieran del aula y se garantizó la confidencialidad (no marcar cuestionario con el nombre). Las encuestas realizadas se recogieron en bolsas negras donde una vez incluidas se revolvían delante de los jóvenes. Se apreció seriedad al contestar con interés en cada pregunta. No todos contestaron la totalidad de las preguntas. Una vez recopilada la información, se hizo un informe para el colegio que fue entregado a orientación escolar.

Manejo estadístico: Se tabuló la información en una hoja Excel y luego se procesaron las frecuencias con el uso del programa Epi Info. De cada pregunta se sumó el total de respuestas obtenidas y se obtuvieron las proporciones de sus correspondientes ítems. Se determinaron los OR y la prueba $p$ de las variables del 2010 versus las obtenidas en 2010.

\section{RESULTADOS}

Tabla 1. Características de la población encuestada en una IED de Bogotá en los años 2010 y 2015

\begin{tabular}{|c|c|c|c|c|}
\hline \multirow{2}{*}{$\begin{array}{l}\text { CARACTERÍSTICAS DE } \\
\text { LA POBLACIÓN }\end{array}$} & \multicolumn{2}{|c|}{2015} & \multicolumn{2}{|c|}{2010} \\
\hline & No & $\%$ & No & $\%$ \\
\hline \multicolumn{5}{|l|}{ Edad } \\
\hline 10 a 12 años & 143 & 19,9 & 63 & 22 \\
\hline 13 a 16 años & 488 & 68,1 & 192 & 66 \\
\hline 17 a 20 años & 86 & 12 & 38 & 13 \\
\hline \multicolumn{5}{|l|}{ Sexo } \\
\hline Masculino & 369 & 51,2 & 142 & 48 \\
\hline Femenino & 352 & 48,8 & 155 & 52 \\
\hline \multicolumn{5}{|l|}{ Estrato } \\
\hline Uno y dos & 80 & 12,1 & 30 & 11 \\
\hline Tres y cuatro & 572 & 86,5 & 253 & 89 \\
\hline Cinco y seis & 9 & 1,4 & 1 & 0 \\
\hline \multicolumn{5}{|l|}{ Estado de ánimo } \\
\hline Triste & 46 & 6,4 & 18 & 6 \\
\hline Normal & 231 & 32,2 & 165 & 56 \\
\hline Feliz & 441 & 61,4 & 114 & 38 \\
\hline \multicolumn{5}{|l|}{ Funcionalidad familiar } \\
\hline Disfunción severa & 25 & 4 & 11 & 4 \\
\hline Disfunción moderada & 145 & 21 & 41 & 14 \\
\hline Funcionalidad normal & 510 & 75 & 232 & 82 \\
\hline \multicolumn{5}{|l|}{ Estructura familiar } \\
\hline Hogar nuclear & 381 & 54 & 158 & 54 \\
\hline Hogar incompleto & 242 & 34 & 89 & 30 \\
\hline Hogar extenso & 49 & 7 & 28 & 9 \\
\hline Hogar reconstituido & 12 & 2 & 1 & 0 \\
\hline Hogar sin padres & 28 & 4 & 19 & 6 \\
\hline
\end{tabular}


Tabla 2. Prevalencias de comportamientos y actitudes relacionadas con el alcohol en adolescentes de una IED de Bogotá, años 2010 y 2015

\begin{tabular}{|c|c|c|c|c|c|c|c|c|}
\hline \multirow{3}{*}{ VARIABLE } & \multicolumn{4}{|c|}{2010} & \multicolumn{4}{|c|}{2015} \\
\hline & \multicolumn{2}{|c|}{ Sí } & \multicolumn{2}{|c|}{ NO } & \multicolumn{2}{|c|}{ sí } & \multicolumn{2}{|c|}{ NO } \\
\hline & No & $\%$ & No & $\%$ & No & $\%$ & No & $\%$ \\
\hline Ha consumido alcohol & 186 & 63 & 109 & 37 & 364 & 54 & 310 & 46 \\
\hline Ha bebido alcohol en la última semana & 102 & 35 & 189 & 65 & 197 & 28 & 507 & 72 \\
\hline Ha tenido dificultades por la forma de beber & 21 & 7 & 279 & 93 & 51 & 7 & 678 & 93 \\
\hline Se ha hecho daño bajo el efecto de alcohol & 11 & 4 & 264 & 96 & 31 & 4 & 744 & 96 \\
\hline Necesita consumir más alcohol cuando bebe & 15 & 5 & 285 & 95 & 26 & 4 & 624 & 96 \\
\hline Padres que beben alcohol con frecuencia & 13 & 4 & 312 & 96 & 32 & 5 & 608 & 95 \\
\hline $\begin{array}{l}\text { Amigos que beben o consumen otras sustancias } \\
\text { psicoactivas con frecuencia }\end{array}$ & 37 & 12 & 271 & 88 & 98 & 14 & 814 & 86 \\
\hline Bebedor frecuente & 104 & 35 & 193 & 65 & 186 & 27 & 503 & 73 \\
\hline
\end{tabular}

Tabla 3. Odds ratios de los comportamientos y actitudes relacionados con el consumo de alcohol en adolescentes de una IED de Bogotá, 2015 versus 2010

\begin{tabular}{|c|c|c|c|c|c|c|c|c|}
\hline \multirow{2}{*}{ VARIABLE } & \multicolumn{2}{|c|}{ SI } & \multicolumn{2}{|c|}{ NO } & \multirow{2}{*}{ OR } & \multirow{2}{*}{$\mathbf{L I}$} & \multirow{2}{*}{ LS } & \multirow{2}{*}{ Prueba p } \\
\hline & No & $\%$ & No & $\%$ & & & & \\
\hline \multicolumn{9}{|l|}{ Ha consumido alcohol } \\
\hline 2015 & 364 & 54 & 310 & 46 & \multirow{2}{*}{0,6881} & \multirow{2}{*}{0,5139} & \multirow{2}{*}{0,9194} & \multirow{2}{*}{0,01093} \\
\hline 2010 & 186 & 63 & 109 & 37 & & & & \\
\hline \multicolumn{9}{|c|}{ Ha bebido alcohol en la última semana } \\
\hline 2015 & 197 & 28 & 507 & 72 & \multirow{2}{*}{0,72} & \multirow{2}{*}{0,5328} & \multirow{2}{*}{0,9757} & \multirow{2}{*}{0,03265} \\
\hline 2010 & 102 & 35 & 189 & 65 & & & & \\
\hline \multicolumn{9}{|c|}{ Ha tenido dificultades por la forma de beber } \\
\hline 2015 & 51 & 7 & 678 & 93 & \multirow{2}{*}{0,9994} & \multirow{2}{*}{0,5778} & \multirow{2}{*}{1,7838} & \multirow{2}{*}{0,8949} \\
\hline 2010 & 21 & 7 & 279 & 93 & & & & \\
\hline \multicolumn{9}{|c|}{ Se ha hecho daño bajo el efecto de alcohol } \\
\hline 2015 & 31 & 4 & 744 & 96 & \multirow{2}{*}{1} & \multirow{2}{*}{0,4809} & \multirow{2}{*}{2,2378} & \multirow{2}{*}{0,8578} \\
\hline 2010 & 11 & 4 & 264 & 96 & & & & \\
\hline \multicolumn{9}{|c|}{ Necesita consumir más alcohol cuando bebe } \\
\hline 2015 & 26 & 4 & 624 & 96 & \multirow{2}{*}{0,7919} & \multirow{2}{*}{0,397} & \multirow{2}{*}{1,6345} & 05938 \\
\hline 2010 & 15 & 5 & 285 & 95 & & & & 0,5938 \\
\hline Padres que beben alcohol co & & & & & & & & \\
\hline 2015 & 32 & 5 & 608 & 95 & & & & \\
\hline 2010 & 13 & 4 & 312 & 96 & 1,2629 & 0,6338 & 2,6611 & 0,5928 \\
\hline Amigos que beben o consum & & & & & & & & \\
\hline sustancias psicoactivas con & & & & & & & & \\
\hline 2015 & 98 & 14 & 602 & 86 & & & & \\
\hline 2010 & 37 & 12 & 271 & 88 & 1,19 & 0,1853 & 1,84 & 0,4515 \\
\hline Bebedor frecuente & & & & & & & & \\
\hline 2015 & 186 & 27 & 503 & 73 & 0.6865 & 0.5078 & 0.93 & 0013 \\
\hline 2010 & 104 & 35 & 193 & 65 & 0,0805 & $0,50 / 8$ & 0,93 & 0,013 \\
\hline
\end{tabular}

Según los resultados encontrados se puede afirmar que en el transcurso de cinco años, de 2010 a 2015, el consumo de alcohol en adolescentes escolarizados ha descendido en un tercio aproximadamente y que esto tiene una significancia estadística importante; asimismo, se observa una disminución en los bebedores frecuentes. Por otro lado, las prevalencias de factores de riesgo como el que los padres consuman alcohol y el que los amigos o compañeras lo hagan, se encontraron iguales, por lo que se podría afirmar que estos dichos factores no están afectando de la misma manera las decisiones en el inicio de consumo y de su mantenimiento. 


\section{DISCUSIÓN}

En este estudio se identificaron algunos sesgos de información relacionados con la seriedad y honestidad de los estudiantes al realizar la encuesta pues, aunque se recalcó que era confidencial y que les permitiría evaluar riesgos, se observó que algunos estudiantes compartían sus encuestas con compañeros lo que indica que pudiera o no ser verdad lo que contestaron; para enfrentar esta situación, las personas que aplicaron la encuesta estaban presentes y se hizo la intervención necesaria para evitar, en lo posible, este sesgo.

Según las respuestas obtenidas en la investigación, el consumo de alcohol por parte de los adolescentes ha disminuido en el período de los cinco años transcurridos (tabla 3). En conformidad con los hallazgos de este trabajo, algunos estudios realizados en Holanda encontraron que el consumo de alcohol en la población juvenil disminuyó al comparar el año 2003 con el 2011 (10) y, en España, se encontró también un descenso, aunque los adolescentes que continúan con el consumo llegan al estado de embriaguez (11). Por su parte, la OMS está mostrando que los países en desarrollo se ven obligados a formular políticas para enfrentar los problemas causados por el abuso del alcohol (12).

¿Cómo se puede explicar la disminución del consumo de alcohol encontrada en este trabajo? Desde el punto de vista gubernamental pudiese ser por las diferentes leyes ${ }^{\ddagger \neq}$ y normas que se han implementado y cuyo objetivo es proteger a la población al prohibir el consumo de alcohol y cigarrillo a los menores de edad $(2,9)$. De igual forma, este descenso se puede explicar por el aumento de los factores protectores tales como la reducción de los mensajes de publicidad§§, políticas de precios, conocimiento de las consecuencias negativas, el seguimiento y la vigilancia en la adolescencia (9).

Según la ley 30 de 1986 en el capítulo III, artículos 14 al 19, se decreta que la venta de bebidas alcohólicas está prohibida para menores de 14 años, cada botella de licor debe tener impresa la etiqueta "el consumo de alcohol es perjudicial para la salud", así como el grado de alcohol que contiene y, además, que el marketing del licor por medios de comunicación debe tener un horario establecido (13). Estas medidas que desarrolló el Gobierno nacional con el fin de prevenir el consumo de alcohol han tenido resultados positivos pues, de acuerdo a Zuleta, el consumo de alcohol ha venido disminuyendo desde 1995 (217 millones de botellas) a 1999 (192 millones de botellas) (14). Ahora bien, teniendo en cuenta el análisis de Gossaín, Colombia es el país de la región más precoz en alcoholismo juvenil debido al fácil acceso al licor por parte de los adolescentes (15) y, si ha disminuido en los últimos cinco años, es porque las estrategias que limitan su acceso están funcionando, como, por ejemplo, el aumento del precio de las bebidas alcohólicas debido a los impuestos de consumo (un aumento de $10 \%$ disminuye del 5 al $12 \%$ el consumo en los jóvenes); asimismo, el control sobre la densidad y ubicación de establecimientos de expendio y la hora de venta, pues cuando los lugares están cerca a la escuela e inician su actividad desde temprano aumenta el consumo (16).

En Colombia, la alcaldía de Bogotá implementó en 113 colegios del Distrito la jornada completa (ocho horas de estudio diarias que van desde las 6:00 a.m. hasta las 3:00 p.m.), brindando a los jóvenes y niños actividades extracurriculares como deportes, artes y la oportunidad de aprender un segundo idioma para que los estudiantes aprovechen todo su tiempo (17). Por otra parte, el Decreto 1355 de 1970 dice que "los reglamentos de la policía local podrán señalar zonas y fijar horarios para el funcionamiento de establecimientos donde se expide alcohol" y, "por motivos de tranquilidad y salubridad pública, los reglamentos de la policía podrán prescribir limitación a la venta de artículos, así como señalar zonas para el establecimiento y el consumo" (18). El Gobierno fomentó, también, una serie de políticas para la protección del adolescente, como las concebidas en el Plan Nacional de Salud Pública, además de estrategias y acciones para identificar y promover la atención y demanda del servicio de rehabilitación y, programas educativos preventivos en medios masivos de comunicación

\#\# http://www.alcaldiabogota.gov.co/sisjur/normas/Norma1.jsp?i=38680

$\S$ http://licoresreyes.es/las-20-mejores-campanas-contra-el-alcohol-al-volante/

Carta Comunitaria. Vol. 23. Número 135. Nov. - Dic. 2015 
para evitar el consumo***. También se ha prohibido el trabajo de menores de edad en establecimientos en donde se produzca, envase, distribuya, expida o se consuma bebidas alcohólicas por parte de los mismos y, para aquellos que sean hallados consumiendo bebidas embriagantes o en estado de embriaguez se ha creado un curso al cual deben asistir con sus padres o acudientes (18).

No se indagó si en el colegio existe o se desarrolló algún programa. Lo cierto es que es esperanzador encontrar que el consumo está disminuyendo.

\section{REFERENCIAS}

(1) Rodríguez D, Dallos M, González S, Sánchez M, Díaz L, Rueda G et al. Asociación entre síntomas depresivos y consumo abusivo de alcohol en estudiantes de Bucaramanga, Colombia [Internet]. Scielo [citado 2005 abril 18]. Disponible en: http://www.scielo.br/pdf/csp/v21n5/12.pdf

(2) Vargas D. Alcoholismo, tabaquismo y sustancias psicoactivas. Rev salud publica [Internet] 2001 [citado 2015 ago 20 ] 3(1): 74-88. Disponible en: http://www.scielo.org.co/pdf/rsap/v3n1/v3n1a06.pdf

(3) Posada J, Herazo E, Campo A. Puerta de entrada al consumo de sustancias ilegales en Colombia: infracción a la norma de incio. Rev unal [Internet] 2009 [citado 2015 ago 2015] 11(3): 406-413. Disponible en: http://www.revistas.unal.edu.co/index.php/revsaludpublica/article/view/37001/39007

(4) Manrique F, Ospina J, García J. Consumo de alcohol y tabaco en escolares y adolescentes de Tunja, Colombia. Rev Salud Pública [Internet]. 2011; 13(1): 89-101. Disponible en: http://www.scielosp.org/pdf/rsap/v13n1/v13n1a08.pdf

(5) Villareal ME, Sánchez JC, Musitu G, Varela R. El consumo de alcohol en adolescentes escolarizados: propuesta de un modelo sociocomunitario. Scielo [Internet] 2010 [citado 2015 ago 2015] 19(3). Disponible en: http://scielo.isciii.es/scielo.php?pid=S1132-05592010000300006\&script=sci_arttext

(6) Álvarez A, Alonso M, Gudorizzi A. Consumo de alcohol y autoestima en adolescentes. [Internet] Latino-Am. 2010; 18: 634-640 [citado 2015 ago 2015]. Disponible en: http://www.scielo.br/scielo.php?script=sci_arttext\&pid=S010411692010000700021

(7) Valderrama H. Pérez Z. Acompañamiento en la adolescencia para prevenir conductas de riesgo. La crianza humanizada: Boletín del grupo de puericultura de la Universidad de Antioquia. 2012; 142: 2-4. Disponible en: http://issuu.com/facultadmedicina/docs/crianza-humanizada-142

(8) Ministerio de Salud de Colombia. Segundo Estudio Nacional de Salud Mental y Consumo de Sustancias Psicoactivas Colombia; 2003. Disponible en: http://onsm.ces.edu.co/uploads/files/1243030_EstudioNacionalSM2003.pdf

(9) Hemphill S, Heerde J, Scholes K, Smith R. Reassessing the effects of early adolescent alcohol use on later antisocial behavior: a longitudinal study of students in Victoria, Australia and Washington state, United States. [Internet]. Early Adolesc. 2014; 34(3): 360-386 [citado 2015 ago 20]. Disponible en: http://www.ncbi.nlm.nih.gov/pmc/articles/PMC4130419/

(10) Verhagen $C$, et al. Does a reduction in alcohol use by Dutch high school students relate to higher use of tobacco and cannabis? Holanda. BMC public health; 2015 [ciado 20 de agosto del 2015] 15:821. Disponible en: http://www.ncbi.nlm.nih.gov/pmc/articles/PMC4549080/pdf/12889_2015_Article_2149.pdf

(11) Sánchez Q, moreno C, Rivera F, Ramos P. Tendencias en el consumo de alcohol en los adolescentes escolarizados españoles a lo largo de la primera década del siglo XXI. España Gac Sanit. 2015; 29(3):184-189, [citado 20 de agosto del 2015]. Disponible en: http://www.gacetasanitaria.org/es/tendencias-el-consumo-alcohollos/articulo/S0213911115000072/

(12) OMS. Estrategia mundial para reducir el consumo nocivo de alcohol [Internet]. 2011[citado 2015 ago 20]. Disponible en: http://www.who.int/substance_abuse/activities/msbalcstrategyes.pdf

(13) Ley 30 de 1986, capítulo III, artículos 14 al 19. Constitución Política de Colombia. Disponible en: http://www.alcaldiabogota.gov.co/sisjur/normas/Norma1.jsp?i=2774

(14) Zuleta L, Jaramillo L. La industria de licores en Colombia. FEDESARROLLO [Internet] (2000) [citado 2015 sept 6]. Disponible en: http://www.fedesarrollo.org.co/wp-content/uploads/2011/08/La-industria-de-licores-en-ColombiaTEXTO-FINAL-CON-TODO.pdf

(15) Gossaín J. Somos el país más precoz de la región en alcoholismo juvenil. EL TIEMPO [Internet] (2012); [citado 2015 Sept 6]. Disponible en: http://www.eltiempo.com/estilo-de-vida/salud/alcoholismo-en-colombia/14871017

(16) Informe de situación regional sobre el alcohol y la salud en Américas. Disponible en: http://www.paho.org/hq/index.php?option=com_docman\&task=cat_view\&gid=1451\&Itemid=270\&lang=es

(17) Bogotá Humana [internet]; 2015 [citado 2015 ago 20]. Disponible en: http://www.bogotahumana.gov.co/EsomeGustadeBogotaHumana/educacion.html

(18) Alcaldía de Bogotá [Internet], Decreto 120 de 2010; [citado 2015 ago 20]. Disponible en: http://www.alcaldiabogota.gov.co/sisjur/normas/Norma1.jsp?i=38680

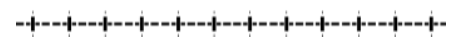

\footnotetext{
${ }^{* * *}$ http://www.saludcapital.gov.co/Lists/Anuncios\%20secundarios/DispForm.aspx?ID=46

Carta Comunitaria. Vol. 23. Número 135. Nov. - Dic. 2015
}

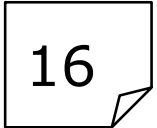

